Table 1 Characteristics of patients with diabetes related autoantibodies after interferon alpha therapy for chronic hepatitis $C$ infection

\begin{tabular}{llrll}
\hline Patient No/sex/age & $\begin{array}{l}\text { Mode of infection/genotype of } \\
\text { HCV }\end{array}$ & $\begin{array}{l}\text { Date of } \\
\text { interferon } \\
\text { therapy }\end{array}$ & $\begin{array}{l}\text { Diabetes related } \\
\text { autoantibodies }\end{array}$ & $\begin{array}{l}\text { Estimated risk } \\
\text { for type 1 } \\
\text { diabetes }\end{array}$ \\
\hline No 1/male/52 y & Unknown/genotype 1 & $8 / 98-8 / 99$ & IAA, GAD 65 & Moderate \\
No 2/male/49 y & IV drug use/genotype 1 & $9 / 98-3 / 99$ & ICA, GAD, IA2 & High \\
No 3/female/48 y & Blood transfusion/genotype 1 & $7 / 98-7 / 99$ & IAA & Low \\
No 4/male/41 y & Unknown/genotype 3 & $11 / 98-5 / 99$ & IAA & Low
\end{tabular}

ICA, islet cell antibodies; GAD65, autoantiodies to glutamate decarboxylase 65; IA2, autoantibodies to tyrosine phosphatase IA2; IAA, insulin autoantibodies.

exclude the existence of any of these autoantibodies prior to interferon alpha therapy.

We identified four patients with diabetes related autoantibodies after cessation of therapy with interferon alpha (table 1). None of the patients had any of these antibodies prior to antiviral therapy nor had they a positive family history for autoimmune diabetes. Patient Nos 3 and 4 only developed insulin autoantibodies at a low titre. Induction of antibodies to insulin is a known phenomenon during therapy with interferon alpha and is described in a frequent number of cases. ${ }^{4}$ Overall, these patients seem to have a low risk of progressing to clinically overt diabetes. Patient No 1 was found to be positive for insulin and GAD65 autoantibodies. Based on prospective clinical studies, this patient has an intermediate risk of developing diabetes. ${ }^{5}$ We have now followed the patient for 16 months after interferon therapy and he has not developed an abnormal fasting glucose so far. The most striking example for induction of diabetes related autoantibodies was found in patient No 2. He developed three major autoantibodies during interferon therapy (GAD65, IA2, and ICA). Based on the predictive value of three positive autoantibody tests, this patient has a considerable risk of developing clinical overt diabetes over the next years. To date (follow up for 12 months) he has not developed an abnormal fasting glucose or an abnormal glucose tolerance test. The situation in this patient is further complicated as he did not respond to the antiviral therapy and another course of interferon might further increase his risk of developing autoimmune diabetes.

In summary, different diabetes related autoantibodies can be induced during interferon therapy for chronic HCV infection. However, we propose that only those patients with more than one autoantibody are at a considerable risk of progressing to clinically overt disease. Therefore, if one autoantibody appears during antiviral therapy, follow up should include screening for all other diabetes related autoantibodies. Furthermore, the question of whether patients with multiple autoantibodies should be retreated with interferon remains unsolved. Here the physician has to weigh possible progression of liver disease against the possibility of inducing autoimmune diabetes.

H E WASMUTH C STOLTE A GEIER

C GARTUNG

S MATERN Medical Department III,

Technical University of Aachen, Germany Correspondence to: H E Wasmuth, Medical Department III, Technical University of Aachen, Pauwelsstraße 30, D-52074 Aachen, Germany. hwasmuth@hotmail.com

1 Schrantz DB, Lernmark A. Immunology in diabetes: an update. Diabetes Metab Rev 1998;14:3-29.
2 McLaren NK, Lan M, Coutant R, et al. Only multiple autoantibodies to islet cells (ICA), insulin, GAD65, IA-2 and IA-2 predict insulin, GAD65, IA-2 and IA-2 predict immune-mediated (type 1) diabetes
tives. F Autoimmun 1999;12:279-87.

3 Wasmuth HE, Scherbaum WA. Autoantibodies in diabetes mellitus: diagnostic value. $\mathcal{F} \mathrm{Lab}$ Med 1999;23:535-42.

4 Betterle C, Fabris P, Zanchetta R, et al. Autoimmunity against pancreatic islets and other tissues before and after interferon-alpha therapy in patients with hepatitis C virus chronic infection. Diabetes Care 2000;23:117781 .

5 Bingley PJ, Bonifacio E, Williams AJK, et al. Prediction of IDDM in the general population. Strategies based on combination of autoantibody markers. Diabetes 1997;46:1701-10.

\section{ERCP training}

EDITOR,- The leading article "ERCP training -time for change" by Hellier and Morris (Gut 2000;47:459-60) addresses important issues. Views as to how endoscopic retrograde cholangiopancreatography (ERCP) training should change may vary considerably between those in the average district general hospital (DGH) serving $220000-250000$, and those in larger units, frequently serving populations of around 500000 .

Firstly, those without ERCP training but additional skills elsewhere will soon find themselves a favoured species. What price a skilled ultrasonographer? Advertising recently for a third consultant gastroenterologist to join two who undertook ERCP, we specified that ERCP was a skill not required. We felt an additional ERCP practitioner would dilute experience and eventually skill. Many DGH trusts are in a similar position with two consultants already in post who can provide sufficient ERCP cover and they do not want a third.

Secondly, the quality of training is largely dependent on two factors: the skill of the trainer, both in relation to ERCP and as an educationalist, and the case exposure available to the trainee. Frequently in a DGH there is only one trainee and case exposure is high. In a larger centre, while the number of ERCPs undertaken may be twice as great, there are frequently $3-5$ trainees wanting to gain experience and "hands on" case experience is unavoidably less.

I am sure that I am not alone in finding that some attached $\mathrm{SpRs}$ have improved rapidly when exposed to a regular weekly list, an exposure that they were unable to achieve at their main teaching centre where teaching was otherwise excellent, simply because of pressure of the number of trainees on lists.

If the final decision is that units undertaking less than an arbitrary number of procedures (currently 250) are not to train SpRs, there are obvious consequences for training beyond further loading of the teaching centres which are already overstretched. It means that if an $\mathrm{SpR}$ is attached to a DGH at a late stage of training, when she is competent to undertake procedures independently, as judged by the main teaching centre, she will be unable to consolidate her skill at the DGH during the attachment. This is because it would be unwise from the clinical governance and medicolegal standpoint for a consultant or trust to allow anyone still defined as being a "trainee" near an ERCP if the unit is not a Joint Advisory Group (JAG) approved training centre.

The top centres in the country have practitioners the skill of which we all admire but few of us working elsewhere could ever approach. They provide excellent live demonstration teaching days and at times informal and friendly one to one advice from which we greatly benefit. Attendance at such live demonstration days should be a required component of all trainees' training and regularly considered for CPD by trainers.

Finally, my recent experience of $\mathrm{SpR}$ applicants for a consultant post had its illuminating aspects. The stated experience of some was such that they had apparently undertaken ERCPs independently on the equivalent entire average clinical load for a DGH for a three year period and this was for a post where ERCP was not required. I did wonder how trainers were maintaining any skill at all.

D BULLIMORE Barnsley DGH Trust, Gawber Road, Barnsley, South Yorkshire S75 2EP, UK dwwbullimore@compuserve.com

\section{Reinnervation after childbirth-a new} paradigm for sensory bowel symptoms?

Visceral hypersensitivity has been identified as a significant feature in a proportion of patients with irritable bowel syndrome. ${ }^{1}$ Reinnervation following a difficult intrapartum episode may be an important contributory factor. Many benign pelvic symptoms may be interpreted as pain or discomfort in response to touch (allodyniae or hyperalgesiae), including chronic pelvic pain, deep dyspareunia, urinary urgency, tampon discomfort, dysmenorrhoea, etc. Premature and prolonged maternal voluntary efforts in the second stage of labour appear to be significant aetiological features in women presenting with these clusters of sensory pelvic symptoms that include laparoscopicallynegative pelvic pain. ${ }^{1}$ Malpresentations, big babies, operative vaginal delivery, and excessive uterine activity may also contribute to the primary visceral denervation. Reinnervation has been demonstrated in the uterus, though an interval of five to ten years precedes the onset of sensory pelvic symptoms. ${ }^{2}$ Similar patterns of reinnervation have been demonstrated in the vulva ${ }^{3}$ and may occur in other pelvic viscera.

Anecdotal reports suggest that women treated with tolterodine tartrate (Detrusitol, Pharmacia, New Jersey) for irritative bladder symptoms, experience some improvement in sensory bowel symptoms - for example, faecal urgency and incomplete emptying. Precise questions about a woman's intrapartum history, medium term reinnervation, and different receptor systems may help to account for the neuropathic hypersensitivity that is such a feature of some forms of irritable bowel syndrome.

MARTIN QUINN

Department of Obstetrics \& Gynaecology, Hinchingbrooke Hospital, Huntingdon, PE29 6NT, UK martin.quinn@hbhc-tr.anglox.nhs.uk 
1 Mayer EA. Some of the challenges in drug development for irritable bowel syndrome. Gut 2001;48:585-6.

2 Quinn MJ, Bourne MR. Levator defects and chronic pelvic pain. American f Obstet Gynecol 2001 (in press).

3 Westrom LV, Willen R. Vestibular nerve fiber proliferation in vulvar vestibulitis syndrome. Obstet Gynecol 1998;91:572-6.

\section{BOOK REVIEWS}

Challenges in Colorectal Cancer. Edited by J H Scholefield (Pp230; illustrated; £69.50). Oxford, UK: Blackwell Science, 2000. ISBN 0632051167 .

If asked to supply a subtitle for this excellent eminently readable book, I would suggest "All you ever wanted to know about the basics of colorectal cancer but were afraid to ask because you assumed that people would expect you to know already". The chapters are written by experts renowned in their field and each topic is dealt with comprehensively and clearly with the reader being led from one line of argument to the next in a satisfyingly logical way. Although aimed at the clinician rather than the scientist, where necessary the scientific evidence is given in sufficient detail without overwhelming the non-expert with technical jargon. Similarly, in this era of evidence based medicine, the authors are to be congratulated on choosing judiciously the most relevant clinical trials which help explain the development of currently accepted clinical practice. Areas of controversy (such as the use of total mesorectal excision (TME) in rectal cancer) are presented in a fair and (largely) unbiased way.

Perhaps a few more figures or diagrams might have been useful, although the easily readable text makes this less of a problem than it might have been (I assume the labelling of the first two figures in the chapter on adjuvant chemotherapy which does not correspond with the text will have been corrected by the time the book is published).

There are a few minor quibbles concerning details of individual chapters. The statement that in rectal cancer the use of TME to reduce local recurrence rates to less than $10 \%$ precludes the need for adjuvant therapy should have read adjuvant radiotherapyadjuvant chemotherapy is still indicated in these patients. The chapter on surgery for recurrent and metastatic disease deals mainly with local recurrence: it would have been interesting to have greater discussion of the role of surgery for hepatic and pulmonary metastases. A short historical introduction to the Dukes' staging system (although well known) and its modifications (perhaps not so well known) would have been appreciated in the chapter on pathology. Some readers may find a little too much technical detail in the otherwise excellent chapter on radiotherapy.

The authors of the chapter on adjuvant chemotherapy may have changed their emphasis on radiotherapy as the "prime adjuvant weapon" in rectal cancer if they had known the results of the recently published
NSABP-R02 trial which showed that radiotherapy seems to be more useful at reducing local recurrence rates (which is probably more of a problem in the subgroup of patients with positive circumferential margins or who undergo suboptimal surgery) rather than improving overall survival: the clinical choice for adjuvant treatment in these patients is probably between chemotherapy alone or combined chemoradiation.

The chapter on future directions misses the opportunity to discuss in greater detail the potential of the new drugs irinotecan, oxaliplatin, and the oral fluoropyrimidines. A separate chapter on imaging techniques might be considered for the next edition, considering the increasing interest in virtual colonoscopy and the value of MRI in helping to determine the resectability of rectal tumours.

I am somewhat loath to mention these points as they are probably a reflection of individual opinion and certainly should not detract from this valuable book, which would be an excellent introduction for a registrar or SHO plunged into the field and expected to be a knowledgeable expert from the outset. Likewise, as the relevance of a multidisciplinary approach to colorectal cancer becomes increasingly important, it is essential that the specialist in one discipline keeps up to date with the current thinking in related specialties: a function which this books serves admirably.

M ALLEN

Surgery of the Anus, Rectum, and Colon, 2nd edn, vols 1 and 2. Edited by M R B Keighley, N S Williams (Pp 2702; illustrated; £285). UK: Harcourt Publishers Ltd, 2000. ISBN 0702023353.

This is a monumental effort and the editors, who have written much of this textbook themselves, are to be congratulated on a terrific job. They have taken on the task of reworking the iconic textbook first started by the late and great John Goligher. His was a very personal style, with repeated references to his own practice and results, and then a weighing of the evidence and a firm opinion, an approach which was much loved. With the growing emphasis on a larger body of evidence, the editors have widened their search in the world's literature and updated it for the now established specialty of coloproctology which tends to be surgical but spans the many disciplines looking after patients with diseases of the lower gastrointestinal tract. And here, despite the view that electronic publishing will consign many textbooks to history, is a second edition, tribute to its success and what Vie Fazio in his foreword calls the perspective "thing". There must still be a place for one or two prominent and authoritative reference textbooks in any specialty provided they are kept up to date, and I have no doubt this will continue to be just such a tome.

There is much here which will be of interest to gastroenterologists as well as surgeons. If you want to argue the point with your local surgeon, give a talk which includes some surgical outcomes, write a medicolegal report, or find a reference, it is all there. The style of the previous edition has been retained, with clear text, excellent line drawings, and helpful tables. The bibliography arranged at the end of each section is exhaustive and comprehensive. New sections have been added on medical management of anal fissure, newer treatments for incontinence, and the combined management of pelvic floor disorders In inflammatory bowel disease, the place of newer therapies have been discussed, and pouch salvage procedures are reviewed. Cancer has been updated with additions to the whole area of biology, natural history, and hereditary and polyposis syndromes, as well as adjuvant therapy, The chapter on laparoscopy in colorectal cancer which includes length of stay and cost issues, anticipates the recent pronouncement of the NICE in concluding that open surgery still has room for improvement, and until specialisation and quality improve these results and there are clear advantages, laparoscopic resection must remain in abeyance outside clinical trials. A good examination of the place of local excision includes transanal endoscopic microsurgery but with so many surgical staff carrying out colonoscopy, the section on technique was rather short and the newer approaches to polypectomy, including endoscopic mucosal resection, and placement of clips and ligatures, and tattooing was disappointing.

Inevitably there are some gaps. Although the place of nitrites is reviewed for the treatment of fissure, the more recent introduction of calcium channel blockers and a growing disenchantment with GTN are missed. In the management of Crohn's fistulae, anti-tumour necrosis factor may have a profound impact on surgical practice but this too did not reach the cut. And the controversial new stapling procedure for haemorrhoids arrived too recently for inclusion.

These are minor criticisms however. Although it comes in two hefty volumes and at a similarly weighty price, this is a must have. It is in my view the finest reference textbook on the subject on both sides of the Atlantic, and will be taken frequently and enjoyably from the bookshelves in offices and studies of those who really care deeply about the management, medical and surgical, of these embarrassing, distressing, and challenging conditions. The owners will just have to make sure the books are returned.

N MORTENSEN

Practical Management of Oesophageal Disease. Edited by A Adam, R Mason, W Owen $(£ 69.95)$. UK: Isis Medical Media Ltd, 2000. ISBN 1-899066-94-2.

The choice of title for a book can make or break its sales. Despite any caution or maturity of judgement we may claim, it seems we are all at risk of being seduced by books with titles such as "Improve your golf", or "Make the stock market work for you". Read the book and find the success/wealth/glamorous lifestyle or whatever that has eluded you up to now. Practical management of oesophageal disease is in this mould - the title hints not at a glamorous lifestyle admittedly, but at delivering the easy answers gastroenterologists would like to find for all oesophageal problems. And of course it does not deliver them. However, if expectations are tempered to the real world rather than fantasy, this is a useful book, written by people who know their subject, describing what they do and why.

In 12 chapters, the 20 authors give their own perspectives on the major topics in oesophageal disease. There is a surgical bias (for example, medical management of gastrooesophageal reflux disease is covered in two 